\title{
Numerical investigation of a one-parameter family of triple close approaches occurring in stellar systems
}

\author{
Victor Szebehely \\ The University of Texas at Austin, Austin, Texas 78712 \\ (Received 2 July 1974)
}

\begin{abstract}
This is the second part of a trilogy dealing with the effect of triple close approaches on the evolution of stellar systems. The first part appeared in the Astron. J. Vol. 79, p. 981 (1974). The results of numerical integrations of stellar systems slightly perturbed from the condition of complete collapse are presented and it is shown that binary formations and escapes occur with systematic regularity and form a well-established family. The time variations of the pertinent parameters of triple close approaches are examined in minute detail. Three different numerical methods of integration are described. The thesis, that triple close approaches are of fundamental importance to the evolution of stellar systems, seems to be supported by the results. The astronomical applications will be offered in the sequel.
\end{abstract}

\section{INTRODUCTION}

$\mathrm{O}$ $\mathrm{NE}$ of the early references by Ambartsumyan (1937) points out the astronomical significance of triple close approaches. Analytical and precise numerical investigation of this phenomenon appears to be essential in the understanding of the dynamic behavior of multiple stellar systems. The study of triple systems instead of two-body systems is more meaningful, but at the same time much more difficult for analytical as well as numerical reasons. The fact that two-body systems with negative total energy are always bounded but triple systems are not, offers one of the important reasons for replacing local two-body considerations with three-body dynamics when gravitational n-body systems are studied. The modest aim here is to study numerically such building blocks formed of three bodies with masses of the same order of magnitude. When an asymmetric triple close approach occurs in such a system, the probability of a binary formation is high, as have been shown by Agekian (1967), Szebehely (1967), and others. According to the Agekian-Szebehely classification of the states of motion in the general problems of three bodies, the family presented in this paper belongs to class $\mathrm{O}$, or "triple close approach." [For these classifications, see Agekian (1973) and Szebehely (1971)].

The analytical aspects of this work were described in a previous paper which appeared in these pages (Szebehely 1974) and which henceforth will be referred to as Paper I.

Only the novice in natural sciences will be surprised by the fact that the physically important phenomenon of triple close approach in three-body dynamics also represents the most severe problem for numerical and analytical treatment. Without dwelling on the generally nonregularizable nature of triple collisions, a theorem usually associated with Siegel (1941) but first discussed by Block (1909), we mention this as the basic reason for the difficulties encountered during numerical integrations of triple configurations. If the total energy of the system is positive, instability always occurs and the system either explodes (all three bodies depart on perturbed hyperbolic orbits) or a binary is formed and the third body escapes.

On the other hand, in the astronomically more important case of negative total energy, one or several triple close approaches proceed an eventual escape, if it occurs at all. Therefore, the detailed behavior during triple close approaches might be considered relevant to stellar dynamics.

\section{DESCRIPTION OF THE SYSTEM}

The analytical description of the initial conditions are given in Paper I and the general arrangement is shown in Fig. 1 of this paper. The three masses are equal, $m_{1}=m_{2}=m_{3}=1$, and the equilateral triangle at $t=0$ has sides of unit length. The initial velocities are selected so that the center of mass is at rest: $v_{1 x}=v_{2 x}$ $=-v_{0} / 2, \quad v_{3 x}=v_{0}, \quad v_{1 y}=v_{2 y}=v_{3 y}=0$. Because of the asymmetry occurring when $v_{0} \neq 0$, the triple collision $\left(v_{0}=0\right)$ is replaced by a triple close approach, provided $v_{0}$ is small (see Paper I). In fact, the moment of inertia of the system, $I=\Sigma m_{i} r_{i}{ }^{2}$, initially is one with $\dot{I}(0)=0$, independently of the value of $v_{0}$. The second derivative initially depends on $v_{0}$ and it is given by $\ddot{I}(0)=3\left(v_{0}{ }^{2}-2\right)$. Consequently, the second derivative at $t=0$ is positive (negative) for $v_{0}>\sqrt{2}$ (for $v_{0}<\sqrt{2}$ ), the curve $I=I(t)$ is convex (concave) from below and the virial coefficient is larger (smaller) than unity. The total energy of the system is conserved and is given by $E_{t}=\frac{3}{4}\left(v_{0}{ }^{2}-4\right)$. Consequently, the energy is positive (negative) if $v_{0}>2$ (if $v_{0}<2$ ). The angular momentum of the system is proportional to $v_{0}$, and since the motion is planar, $c=\sqrt{3} v_{0} / 2$. The arrangement shows symmetry with respect to the $y$ axis (Fig. 1), therefore, only positive values of $v_{0}$ are considered. For small values of $v_{0}$ the total energy and $\ddot{I}(0)$ are negative and the virial coefficient is less than one. This is certainly true for $0 \leq v_{0}<\sqrt{2}$, consequently in this range the motion begins with a contraction.

The three papers forming the trilogy are restricted to low initial velocities, i.e., $0<v_{0} \leq 0.2$ since their 


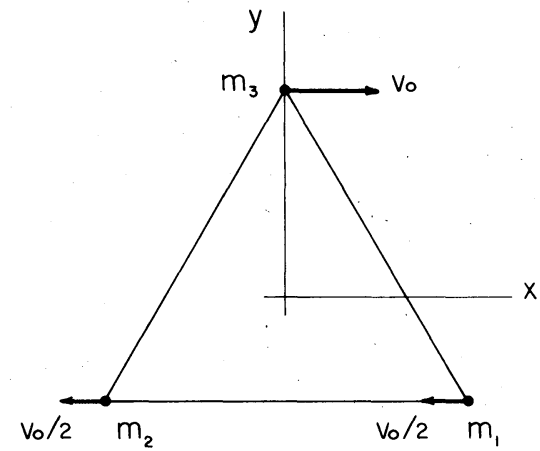

Fig. 1. Initial conditions.

purpose is to investigate triple close approaches and the subsequent binary formations. Escapes of $m_{2}$ and associated binary formations occur in the range of $0<v_{0} \leq 0.186$. As the initial velocity increases to $v_{0} \geq 0.187$ the escape changes into the ejection of $m_{2}$ and eventually as $v_{0}$ further increases, the mode becomes what is known as interplay. The global behavior of the system, i.e., the study of the range $0.2<v_{0}$ will be presented elsewhere.

The choice of the initial configurations and initial velocities is governed by the simplicity of treatment, usefulness of showing the prominent physical aspects, and applicability to stellar dynamics. In our case the model clearly shows the effect of small initial velocities, the role of triple close approaches are emphasized, the parameterization is natural, and the numerical values found are realistic. This last item will become more apparent in the third part of the trilogy.

\section{RESULTS OF NUMERICAL INTEGRATIONS}

\section{A. Analysis of the Behavior for $v_{0}=0.001$}

The initial velocity is varied from $v_{0}=10^{-10}$ to $v_{0}=0.2$ and the trajectories of the particles are obtained by numerical integration for each case using $G=1$, for the constant of gravity. The second body escapes (or is ejected) in all cases. The asymptotic hyperbolic escape velocity $v_{\infty}$, the semimajor axis of the binary formed $a_{\infty}$, its eccentricity $e$, the direction of escape, the minimum moment of inertia $I_{m}$, the time $t_{m}$ when it is reached, the close binary approaches and the conditions for escape are evaluated for all cases. When escape does not occur, the elliptic ejection path is established. All escapes were verified by Standish's (1971) escape criteria.

A typical, representative member of the family with $v_{0}=0.001$ is discussed in some detail in the following. Figure 2 shows the critical part of the triple close approach. Because of the small initial velocities, the three bodies begin their motion with a contraction toward the origin where the center of mass is located. The system will collapse (with zero initial velocity) in $t_{c}=\pi /(24)^{\frac{1}{2}}$ time units as shown in Paper I. (Translation

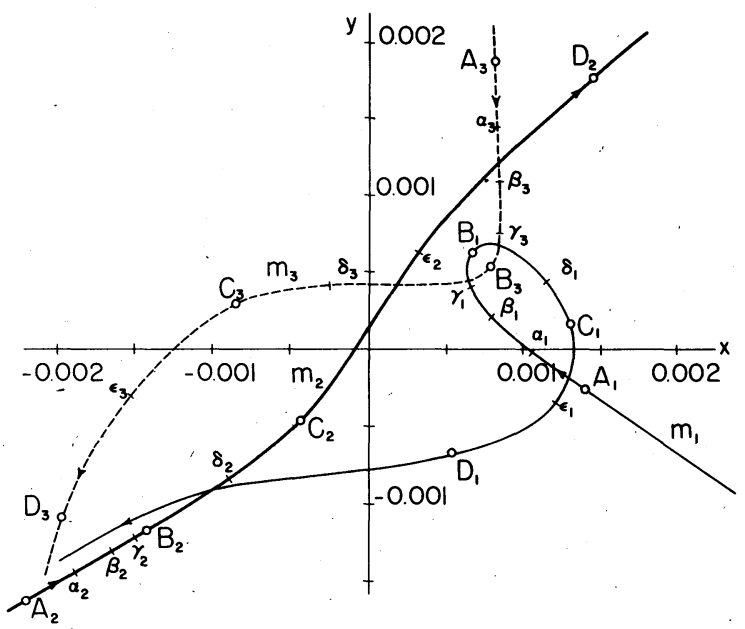

FIG. 2. Orbits near triple close approach $\left(v_{0}=0.001\right)$.

of the dimensionless quantities to physically significant values will be described at the end of the paper and in the sequel.) The corresponding time with nonzero initial velocity might be taken to be when the quantity $\Sigma r_{i j}{ }^{2}$ reaches its minimum. Since the moment of inertia with respect to the center of mass is

$$
I=\frac{1}{M} \sum m_{i} m_{j} r_{i j}{ }^{2}
$$

where $1 \leq i<j \leq 3$, we have for equal and unit masses,

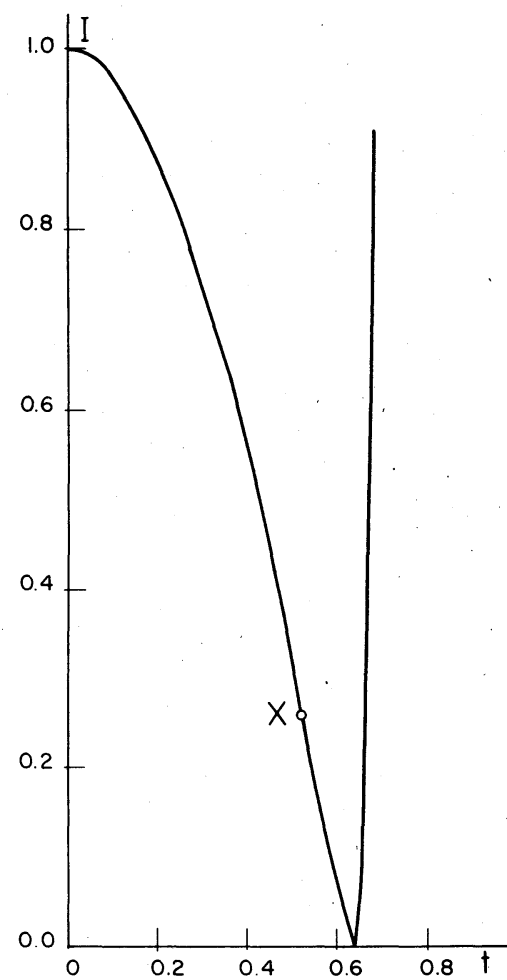

Fig. 3. Moment of inertia versus time $\left(v_{0}=0.001\right)$. 
TABLE I. Times corresponding to the points shown on the orbits in Fig. 1.

\begin{tabular}{|c|c|c|c|c|c|c|c|c|c|}
\hline Point & $A_{i}$ & $\alpha_{i}$ & $\beta_{i}$ & $\gamma_{i}$ & $B_{i}$ & $\delta_{i}$ & $C_{i}$ & $E_{i}$ & $D_{i}$ \\
\hline $\begin{array}{l}\text { Time } \\
\tau\end{array}$ & $\begin{array}{c}0.641218 \\
218\end{array}$ & $\begin{array}{c}0.641234 \\
234\end{array}$ & $\begin{array}{c}0.641244 \\
244\end{array}$ & $\begin{array}{c}0.641250 \\
250\end{array}$ & $\begin{array}{c}0.641254 \\
254\end{array}$ & $\begin{array}{c}0.641274 \\
274\end{array}$ & $\begin{array}{c}0.641288 \\
288\end{array}$ & $\begin{array}{c}0.641316 \\
316\end{array}$ & $\begin{array}{c}0.641356 \\
356\end{array}$ \\
\hline
\end{tabular}

that $I=\frac{1}{3} \Sigma r_{i j}{ }^{2}$. Consequently, the time necessary to reach the minimum value of the moment of inertia might be compared to the above collapse time $t_{c}=0.6412749151$.

The asymmetry is apparent when the bodies are at the points marked $A_{i}$ in Fig. 2. The next interesting feature occurs when $m_{1}$ and $m_{3}$ experience a close approach (see points $B_{1}$ and $B_{3}$ ). At this time $m_{2}$ is delayed because of the directions of the initial velocities. In fact, $m_{2}$ is a latecomer for all members of the family of orbits under discussion and, consequently, it is always the escaping or the ejected body. This first close binary approach, $r_{13}=1.5626 \times 10^{-4}$ occurs at $t_{13}=0.641254$ and the dimensionless relative velocity between $m_{1}$ and $m_{3}$ is $v_{13} \cong 160$ velocity unit. The second body at the same time is about twice as far from the origin as $m_{3}$ and $m_{1}$ and the moment of inertia of the system is $5.134 \times 10^{-6}$. This is not the value for $I_{\min }$, since $m_{2}$ and $m_{3}$ still move toward the center of mass (while $m_{1}$ is in a relatively slow loop-orbit). The second body increases its velocity between points $B_{2}$ and $C_{2}$ since both $m_{1}$ and $m_{3}$ attract it. At $t_{m}=0.641288$ a close approach occurs between $m_{2}$ and $m_{3}, r_{23}=8.64 \times 10^{-4}$, and at the same time $I_{\min }=2.919 \times 10^{-6}$ is established (see points $C_{i}$ ). The relative velocity during this close approach is $v_{23} \cong 88$. The velocity of the first body $m_{1}$, at the same time, is only about $v_{1} \cong 20$ units, and it is moving about normal to the directions of $m_{2}$ and $m_{3}$, which latter bodies move with $v_{2} \cong 47$ and $v_{3} \cong 41$ (see Fig. 2). The distances from $m_{1}$ to $m_{2}$ and $m_{3}$ are about $2 r_{23}$. The three distances from the center of mass at

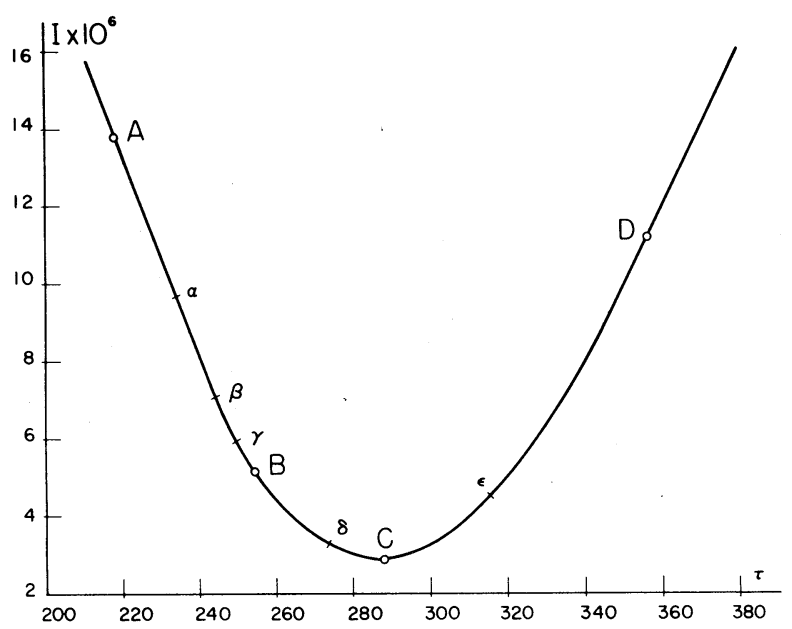

Fig. 4. Moment of inertia at triple close approach $\left(v_{0}=0.001\right)$. $t_{m}$ are $r_{1}=13.0 \times 10^{-4}, r_{2}=6.4 \times 10^{-4}$, and $r_{3}=9.0 \times 10^{-4}$. After this time $m_{2}$ escapes, and $m_{1}$ and $m_{3}$ form a binary. The escape is a consequence of the asymmetric initial conditions as well as of three-body effects. Without $m_{1}$ 's presence, $m_{2}$ and $m_{3}$ would have formed a higheccentricity binary and no escape could have occurred since the total energy of the system is negative, forbidding unbounded two-body orbits.

The additional marks on the orbits $\left(\alpha_{i}, \beta_{i}, \gamma_{i}, \delta_{i}\right.$, and $\left.\epsilon_{i}\right)$ are synchronized. The times corresponding to the 27 points of Fig. 2 are listed in Table I. In what follows, the quantity $\tau$ will be used together with the time $t$. The relation is $t=0.641+\tau \times 10^{-6}$.

The variation of the moment of inertia with time is shown in Fig. 3 and 4 and $\dot{I}=\dot{I}(t)$ is presented in Fig. 5. Note the expected smoothness of $I(t)$ in the magnified plot of Fig. 4, the sharp rise after $I_{\min }$ in Fig. 3, and the asymmetric behavior of $\dot{I}(t)$ in Fig. 5 . The largest change in $\dot{I}$ occurs at point $B$ when $\ddot{I}$ is maximum (see also Fig. 7) due to the close approach of $m_{1}$ and $m_{3}$.

The virial coefficient $\alpha=2 T / F \geq 0$ and the total energy $E_{t}=T-F<0$ may be used to express the kinetic energy, $T=E_{t} \alpha(\alpha-2)^{-1} \geq 0$, the negative of the potential energy, $F=2 E_{t}(\alpha-2)^{-1} \geq 0$, and the second derivative of the moment of inertia, $\ddot{I}=4 E_{t}(\alpha-1)(\alpha-2)^{-1}$. Since the total energy is negative for the family under discussion, we have

$$
T=\frac{\left|E_{t}\right| \alpha}{2-\alpha}, \quad F=\frac{2\left|E_{t}\right|}{2-\alpha}, \quad \ddot{I}=\frac{4\left|E_{t}\right|(\alpha-1)}{2-\alpha} .
$$

During the motion every member of the family has a fixed $E_{t}$ value and $T, F$, and $\ddot{I}$ change with $\alpha$ as $\alpha$ varies with time. It may be shown that $\alpha \leq 2$, the equality corresponding to a binary collision, while

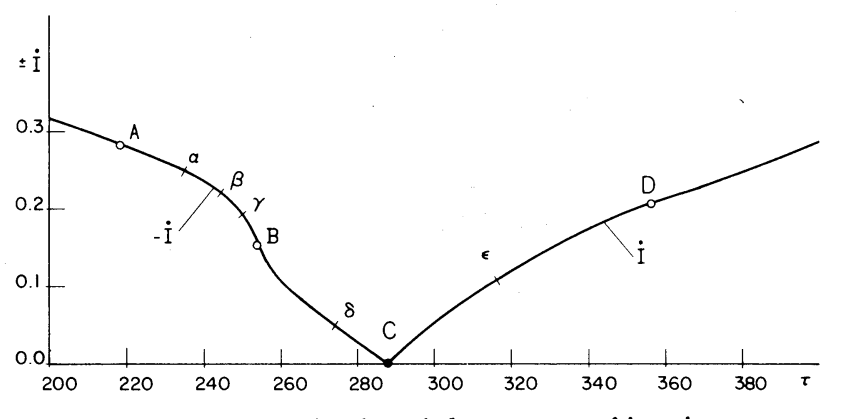

FIG. 5. First derivative of the moment of inertia at triple close approach $\left(v_{0}=0.001\right)$. 
I

2

I্য

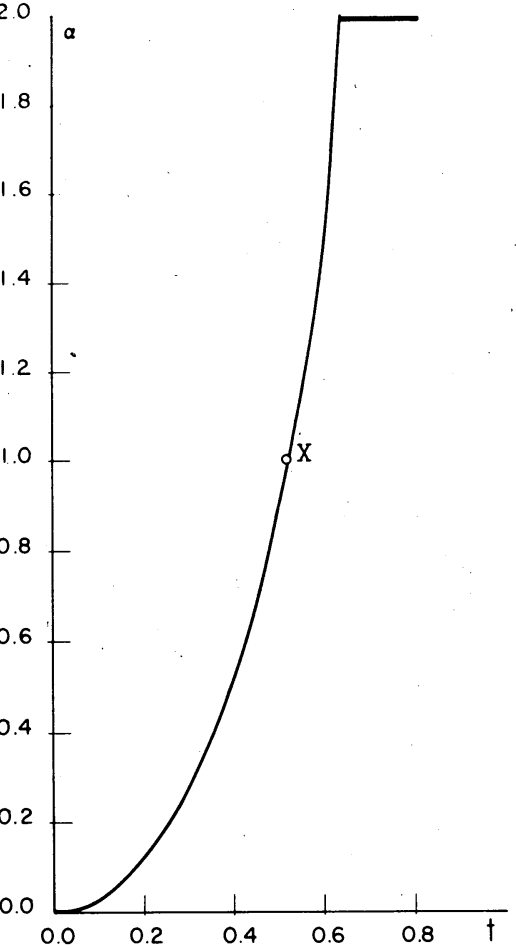

$\alpha=1$ corresponds to $\ddot{I}=0$ as also follows from the Lagrange-Jacobi equation.

Figures 6 and 7 show the variation of the virial coefficient with time for $v_{0}=0.001$. At $t=0$ the value of the virial coefficient is $\alpha=v_{0}^{2} / 2=5 \times 10^{-7}$. At $t \cong 0.52$ the $I(t)$ curve has an inflection point and $\alpha=1$ (see point $X$ in Figs. 3 and 6 ). At the first close binary approach (between $m_{1}$ and $m_{3}$ ) $\alpha=1.99916$ as shown in Fig. 7, point $B$. Because of the compressed time scale in Fig. 6, no details can be seen. The high-frequency variation of $\alpha$ beginning at $t=0.64$ in Fig. 6 corresponds to the final binary formed between $m_{1}$ and $m_{3}$. The period of this binary is $T \cong 3 \times 10^{-4}$, corresponding to $a \cong 1.6746 \times 10^{-3}$. The details may be seen in Fig. 7, where the letters correspond to the points shown in Fig. 2. Attention is directed to point $B$ at the absolute maximum of the curve $\alpha(t)$. At this point the absolute value of the potential is maximum. At point $C$ the moment of inertia is minimum and $\alpha$ has another maximum, smaller. than at point $B$. Consequently, $F_{B}>F_{C}$, and therefore, the minimum value of $I$ is not associated with the maximum of $F$. The value of $F$ may be governed by one single close binary approach while $I$ is governed by the closeness of all three bodies. This fact agrees with Agekyan's (1969) observations and following him we introduce as a measure of the probability of escape $\eta=F /\left|E_{t}\right|=(1-\alpha / 2)^{-1}$ computed when $I_{\min }$ occurs. At point $C$, when the minimum moment of inertia occurs, $\eta=722$. This value is due to a close triple approach, which is indeed needed for escape and which is an indication of a forthcoming escape. At point $B$ the close binary approach gives $\eta=2373$. In comparison, if the initial velocity is $v_{0}=0.2$ (and ejection occurs instead of escape) the critical value of the virial coeffcient at $I_{m}$ is $\alpha=1.8$ and Agekian's measure becomes $\eta=10$.

After point $D$, the curve in Fig. 7 reaches a minimum, rises again, and will oscillate because of the binary formation as mentioned before.

\section{B. Characteristics of the Family}

As the initial velocity changes from $10^{-1}$ to $10^{-10}$ the previously described numerical values change, but not the shape of the orbits. We first discuss the behavior of the close binary approach $r_{23}{ }^{*}$ at $t_{m}$, i.e., at the time when $I_{\min }$ occurs. Figure 8 shows that in the range $10^{-2} \leq v_{0} \leq 10^{-10}$ the function $r_{23}{ }^{*}\left(v_{0}\right)$ is linear on a $\log -\log$ scale, i.e., $r_{23}{ }^{*}=A v_{0}{ }^{n}$ with $A \cong 0.38$ and $n \cong 0.87$.

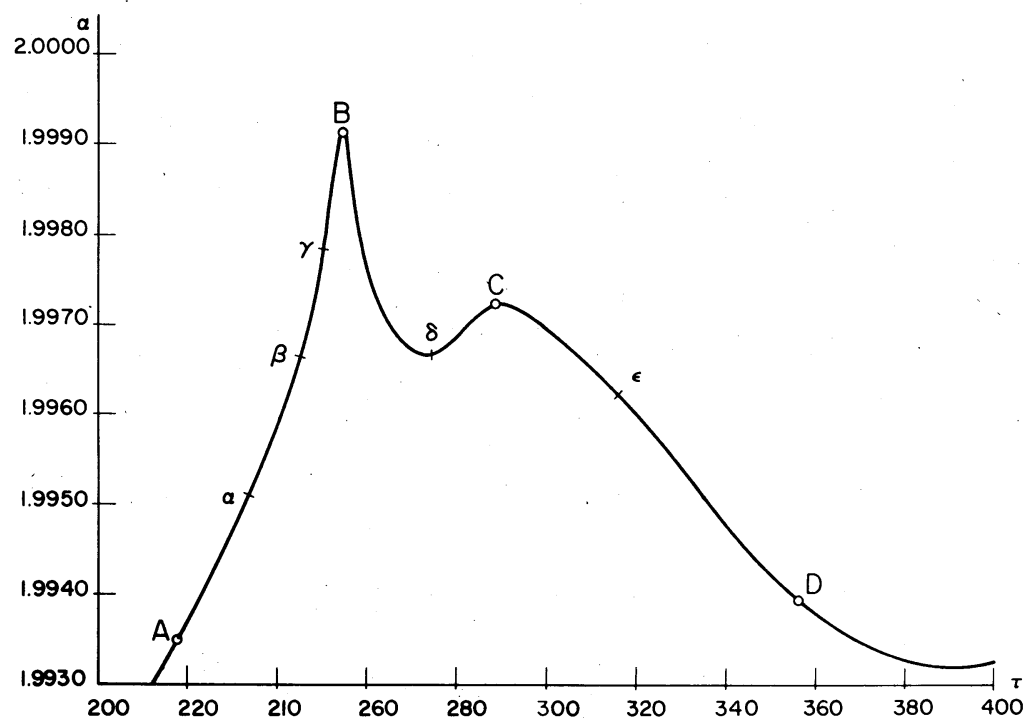

Fig. 7. Virial coefficient at triple close approach $\left(v_{0}=0.001\right)$. 
As the initial velocity becomes larger, the curve flattens out and the above relation is not valid. In fact, while the topology of the orbits remains the same in the complete range of the investigation, the role of the two close binary approaches, $r_{23}{ }^{*}$ and $r_{13}{ }^{*}$, changes at approximately $v_{0}=10^{-1}$. The first close binary approach is always between $m_{1}$ and $m_{3}$ and the second between $m_{2}$ and $m_{3}$, but $r_{12}{ }^{*}$ is smaller than $r_{23}{ }^{*}$ only in the range $10^{-2} \leq v_{0} \leq 10^{-10}$.

In the same range we find the following inequalities persisting:

$$
a_{\infty} \geq\left(I_{m} / 3\right)^{\frac{1}{2}} \geq r_{23} * \geq a_{\infty}(1-e) \geq r_{13} *
$$

It is essential to realize that only two of the quantities shown in this inequality occur simultaneously. The minimum moment of inertia $I_{m}$ occurs at $t_{m}$, simultaneously with $r_{23}{ }^{*}$, but $r_{13}{ }^{*}$ occurs earlier and the binary is formed later. The quantity $\left(I_{m} / 3\right)^{\frac{1}{2}}$ is also known as the radius of gyration, the total mass being three. This quantity is used by Birkhoff (1927) and Sundman (1912) instead of the moment of inertia.

The numerical results not only give the above inequalities, but also show that the ratios of the quantities involved are approximately constant in the (more limited) range of $10^{-3} \geq v_{0} \geq 10^{-10}$. Dividing by $r_{23} *$ we have

$$
\begin{aligned}
\frac{a_{\infty}}{r_{23} *} \cong 1.818 \geq \frac{\left(I_{m} / 3\right)^{\frac{1}{2}}}{r_{23} *} \cong 1.055 \geq 1 \geq & \frac{a_{\infty}(1-e)}{r_{23} *} \\
& \cong 0.727 \geq \frac{r_{13} *}{r_{23} *} \cong 0.154 .
\end{aligned}
$$

From this it follows that the eccentricity is the same for all members of the family, $e=0.6$.

Regarding the escape velocity generated, $v_{\infty}$, it was shown in Paper I that in the limit $v_{\infty}^{2} a_{\infty} \rightarrow \frac{2}{3}$ as $v_{0} \rightarrow 0^{+}$ and, in general, for the members of the family,

$$
v_{\infty}^{2}=2\left(3 a_{\infty}\right)^{-1}+v_{0}^{2}-4 .
$$

This relation shows good agreement with the numerical results in the (less limited) range of $0.186 \geq v_{0} \geq 10^{-10}$. Figure 8 shows the escape velocities versus the parameter $v_{0}$ when $v_{0} \leq 10^{-2}, a_{\infty} \leq 0.012, v_{\infty} \geq 7.18$, and the above equation is already close to its limiting form. The slope of the line $r_{23} *\left(v_{0}\right)$ is twice that of the line $v_{\infty}\left(v_{0}\right)$ in these $\log -\log$ presentations, since $v_{\infty}^{2} r_{23}{ }^{*}=v_{\infty}{ }^{2} a_{\infty} / 1.818$ $\cong 0.3667$ for $v_{0} \leq 10^{-2}$.

The maximum velocity between $m_{1}$ and $m_{3}$ occurring at their close approach $v_{13}$ is related to the escape velocity according to the numerical results by $v_{13}=8 v_{\infty}$ in the range $10^{-2} \leq v_{0} \leq 10^{-10}$.

The general shape of the orbits is the same for all members of the family as mentioned before, but some interesting details change. The direction of the escape of $m_{2}$ is along the positive $y$ axis when $v_{0} \cong 0.08$, and the angle decreases with decreasing $v_{0}$, approaching $30^{\circ}$

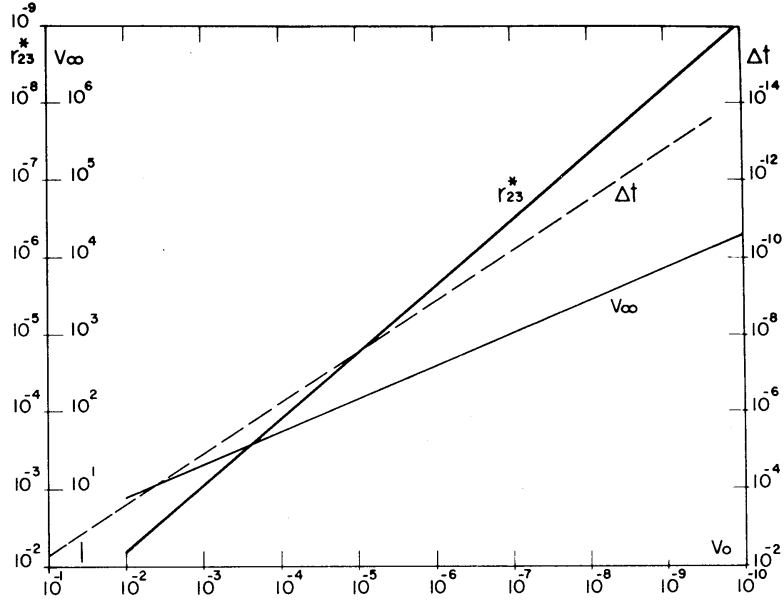

FIG. 8. Distance at the first close binary approach $r_{23}{ }^{*}$, escape velocity $v_{\infty}$, and time to triple close approach $\Delta t$, vs the parameter $v_{0}$.

as $v_{0} \rightarrow 0$. When $v_{0}$ is high enough to prevent escape, ejection occurs along the negative $x$ axis. This change of the direction of departure of $m_{2}$ occurs in a continuous manner.

The final characteristics analyzed for the family is the time $t_{m}$ to reach $I_{\mathrm{min}}$. Introducing $\Delta t=t_{m}-t_{c} \geq 0$, where $t_{c}=\pi /(24)^{\frac{1}{2}}$ as mentioned before, we may show the monotonically increasing function $\Delta t\left(v_{0}\right)$ in Fig. 8.

\section{NUMERICAL METHODS USED}

Most of the members of the family were established by three different computer programs using the University of Texas CDC-6600. The purpose was not to compare the efficiency of the various numerical techniques applied, but rather to obtain consistent and reliable numerical results.

The numerical integration was performed in the first program by a 15th-degree polynomial using Steffenson's (1956) method. Levi-Civita's (1903) two-dimensional regularization was applied to the smallest of the three distances at all times. This program is described by Szebehely and Peters (1967). The second program used a Runge-Kutta-Fehlberg 7(8) integrator with the Kustaanheimo-Stiefel (1965) regularization. For details of this program see Szebehely and Bettis (1971). The third program employed the same integrator as the second with the Aarseth-Zare (1974) regularization applied simultaneously to the two smallest distances at all times.

The major problem in the numerical integration is the proper treatment of the triple close approaches since even binary collisions can be regularized without difficulty. When $v_{0}=10^{-10}$, the smallest distance $r_{12}{ }^{*}$ encountered during the first close binary approach is of the order of $10^{-10}$. The second close approach $r_{23}{ }^{*}$ occurring with $I_{\min }$ is of the order of $10^{-9}$ and the other two distances at the same time are of the same 
order of magnitude. The programs were able to handle this case $\left(v_{0}=10^{-10}\right)$ without difficulty. In fact, the Aarseth-Zare program failed only at $v_{0}=10^{-13}$, which was expected since a 14-digit single-precision arithmetic was used.

As long as the initial velocity was larger than $10^{-11}$, the total energy was preserved to one part in a thousand during the numerical integration. It is a characteristic phenomenon occurring in such problems that the computation of the energy constant may contain significant errors since it uses actual (nonregularized quantities) while the integration is performed using regularized variables. In other words, the orbit is computed with high accuracy in the regularized system, but various transformed printouts using physical (nonregularized) variables might be in error. This explains the spurious fact that the energy constant "recovers" after close approaches. The well-known dictum and principle of Ellis Strömgren regarding the accuracy of periodic orbits in the Copenhagen problem is applicable to our case, i.e., all orbits are computed with high enough accuracy to establish the family.

\section{CONCLUDING REMA:RKS AND APPLICATIONS}

This paper, the second of three, is dedicated to the numerical aspects of triple close approaches. All results are presented in nondimensional form to facilitate any applications. The nondimensional parameter is $G M T^{2} L^{-3}=1$ in the numerical work. Here, $G$ is the constant of gravity and $M, T, L$ are the units of mass, time, and length. The third paper of the trilogy will discuss the applications of this work to stellar dynamics. At this point an example might be mentioned concerning the case of $v_{0}=0.001$ which is treated in considerable detail in the text. For characteristic units we use $L=1 \mathrm{pc}$ and $M=M_{\odot}$ giving $T \simeq 1.5 \times 10^{7} y$, and $V$ $=0.0655 \mathrm{~km} / \mathrm{sec}$ for the unit of time and unit of velocity. At the first close approach $r_{13}{ }^{*} \cong 1.6 \times 10^{-4}=32$ $\mathrm{AU}=6400 R_{\odot}$, and the relative velocity is $v_{13}=160=10.5$ $\mathrm{km} / \mathrm{sec}$. The minimum moment of inertia is reached in $t_{m} \cong 0.64129=9.6 \times 10^{6} y$ from the beginning of the motion when the three Sun-like stars are $1 \mathrm{pc}$ apart with initial velocities of the order of $0.1 \mathrm{~m} / \mathrm{sec}$. The binary formed has a semimajor axis $a_{\infty} \cong 1.9 \times 10^{-3}=380 \mathrm{AU}$ and eccentricity $e \cong 0.5$ with periastron distance of $a_{\infty}(1-e) \cong 186 \mathrm{AU}$. The escape velocity is $v_{\infty} \cong 20 \cong 1.3$ $\mathrm{km} / \mathrm{sec}$.

\section{ACKNOWLEDGMENTS}

The computations were assisted by my students J. Dunham, L. Kruczynski, and K. Zare. A grant from the Astronomy Division of the National Science Foundation made this work possible. I wish to thank Dr. R. Harrington and Dr. E. M. Standish for their comments.

\section{REFERENCES}

Aarseth, S., and Zare, K. (1974). Celest. Mech. 10, 217.

Agekian, T. A., and Anasova, Zh. P. (1967). Astron. Zh. 44, 1261.

Agekian, T. A., Anasova, Zh. P. and Bezgubova, V. N. (1969). Astrofizika 5, 637.

Agekian, T. A., and Martinova, A. I. (1973). Univ. of Leningrad Publ., No. 1, 122.

Ambartsumyan, V. A. (1937). Astron. Zh. 14, 3.

Birkhoff, G. D. (1927). Dynamical Systems, Am. Math. Soc. Publ., Providence, R. I.

Block, H. (1909). Medd. Lunds Astron. Obs. Series 2, No. 6. Kustaanheimo, P., and Stiefel, E. (1965). J. Reine Angew, Math. 218, 204.

Levi-Civita, T. (1903). Ann. Math. [3] 9, 1.

Siegel, C. L. (1941). Ann. Math. 42, 127.

Standish, M. (1971). Celest. Mech. 4, 44.

Steffensen, J. F. (1956). Kgl. Danske Videnskab. Selskab, Mat-Fys. Medd. 30, No. 18.

Sundman, K. F. (1912). Acta Math. 36, 105.

Szebehely, V., and Peters, F. (1967). Astron. J. 72, 876.

Szebehely, V. (1971). Celest. Mech. 4, 116.

Szebehely, V., and Bettis, D. G. (1971). Astrophys. Space Sci. $13,365$.

Szebehely, V. (1974). Astron. J. 79, 981. Paper I. 